\title{
Selection of climate policies under the uncertainties in the Fifth Assessment Report of the IPCC
}

\author{
L. Drouet ${ }^{1,2 \star}$, V. Bosetti ${ }^{1,2,3}$ and M. Tavoni ${ }^{1,2,4}$
}

\begin{abstract}
Strategies for dealing with climate change must incorporate and quantify all the relevant uncertainties, and be designed to manage the resulting risks ${ }^{1}$. Here we employ the best available knowledge so far, summarized by the three working groups of the Fifth Assessment Report of the Intergovernmental Panel on Climate Change (IPCC AR5; refs 2-4), to quantify the uncertainty of mitigation costs, climate change dynamics, and economic damage for alternative carbon budgets. We rank climate policies according to different decision-making criteria concerning uncertainty, risk aversion and intertemporal preferences. Our findings show that preferences over uncertainties are as important as the choice of the widely discussed time discount factor. Climate policies consistent with limiting warming to $2^{\circ} \mathrm{C}$ above preindustrial levels are compatible with a subset of decision-making criteria and some model parametrizations, but not with the commonly adopted expected utility framework.
\end{abstract}

Many of the uncertainties surrounding climate change are difficult to quantify and depend on the judgement of experts and on the type of model used to generate future scenarios. Each model produces a distribution over the possible states of nature (for example, cost of mitigation, temperature increase, or economic damage from climate change), and these distributions might differ from model to model. How should we select climate policy in the face of these uncertainties?

This paper addresses this question using a framework that accounts for both state uncertainty (for example, the distribution over states of nature) and model uncertainty (for example, the different models (or experts) which generate distributions over states $)^{5}$. We investigate a variety of preferences and assumptions over these two types of uncertainty. A special case is the subjective expected utility ${ }^{6}$ framework, traditionally used in economic evaluations. However, an expected utility setting might not work when the information is incomplete and ambiguous, which is clearly the case for climate change ${ }^{7}$. Moreover, people are known to approach risks and uncertainties differently ${ }^{8}$. The proposed setting allows us to explore additional decision-making criteria to deal with uncertainty, in the spirit of refs 7,9. Alternative statistical techniques, consistent with Bayesian approaches, have been developed to cope with model uncertainty ${ }^{10}$. Model weighting is an active topic in climate research ${ }^{11}$, where historical observations provide a basis for model evaluation, although it is not commonly used ${ }^{12}$. Although our framework is sufficiently flexible to accommodate different prior probability measure over the set of possible models, our baseline model assumes a uniform prior with equal model weights.
The literature on the role of uncertainty in climate policy making has mostly relied on either analytical or simplified integrated assessment models (IAMs), such as DICE (ref. 13). In such contexts, different decision-making criteria and preferences over risks have been shown to have a significant impact on the optimal abatement strategy ${ }^{14,15}$. However, these exercises lack detail in the representation of the mitigation options and the climate dynamics. Larger-scale models, which capture the main interrelationships between human and natural systems, have incorporated uncertainty only partially owing to computational limitations. Therefore, uncertainty is mostly treated by means of multi-model ensembles ${ }^{16,17}$, or by single models performing Monte Carlo simulations ${ }^{18,19}$. When accounting for all the key sources of uncertainty, the selection of optimal climate policy has been shown to be more sensitive to uncertainty about mitigation costs and impacts than to uncertainty about warming ${ }^{20}$.

Figure 1 illustrates our approach. Using the best available knowledge from the three working groups of IPCC AR5 (see Methods), for each component (mitigation costs, temperature and climate damage) we generate probability estimates for different classes of models. The decision variable is the carbon budgetthat is, the cumulative $\mathrm{CO}_{2}$ emissions over the twenty-first century (2010-2100). Carbon budgets are robust policy indicators, as they are strictly related to global warming ${ }^{21}$ and climate targets ${ }^{22}$. We assume that uncertainty resolves immediately, but show that our results are robust to different timings of resolution of uncertainty (in Supplementary Fig. 12).

We extract emission projections and associated mitigation costs from the AR5 WGIII Scenario database ${ }^{4}$, which includes the outcomes of many long-term scenarios produced by the most well-established IAMs. The database spans a wide range of policy stringency, and thus of associated carbon budgets, covering the whole range of the representative concentration pathways. The relationship between mitigation costs, harmonized across different metrics, and carbon budgets is found to be nonlinear and highly uncertain (Supplementary Fig. 2). Furthermore, the uncertainty of mitigation costs increases with time (Supplementary Fig. 3). From Supplementary Fig. 2 emerges a well-documented ${ }^{4}$ distinction between different classes of IAMs: top-down (TD) models provide a more accurate description of the economy, whereas bottom-up (BU) models better represent the set of mitigation technologies. TD models generally show higher mitigation costs than BU models, but it is not obvious which class is the most reliable. We account for this model type uncertainty by estimating different probabilistic models of the evolution of mitigation costs.

\footnotetext{
${ }^{1}$ Fondazione Eni Enrico Mattei (FEEM), Corso Magenta 63, 20123 Milan, Italy. ${ }^{2}$ Centro Euro-mediterraneo sui Cambiamenti Climatici (CMCC), Corso Magenta 63, 20123 Milan, Italy. ${ }^{3}$ Bocconi University, Department of Economics, Via Sarfatti 25, 20136 Milan, Italy. ${ }^{4}$ Politecnico di Milano, Department of Management and Economics, Via Lambruschini 4/B, 20156 Milan, Italy. ${ }^{\star}$ e-mail: laurent.drouet@feem.it
} 


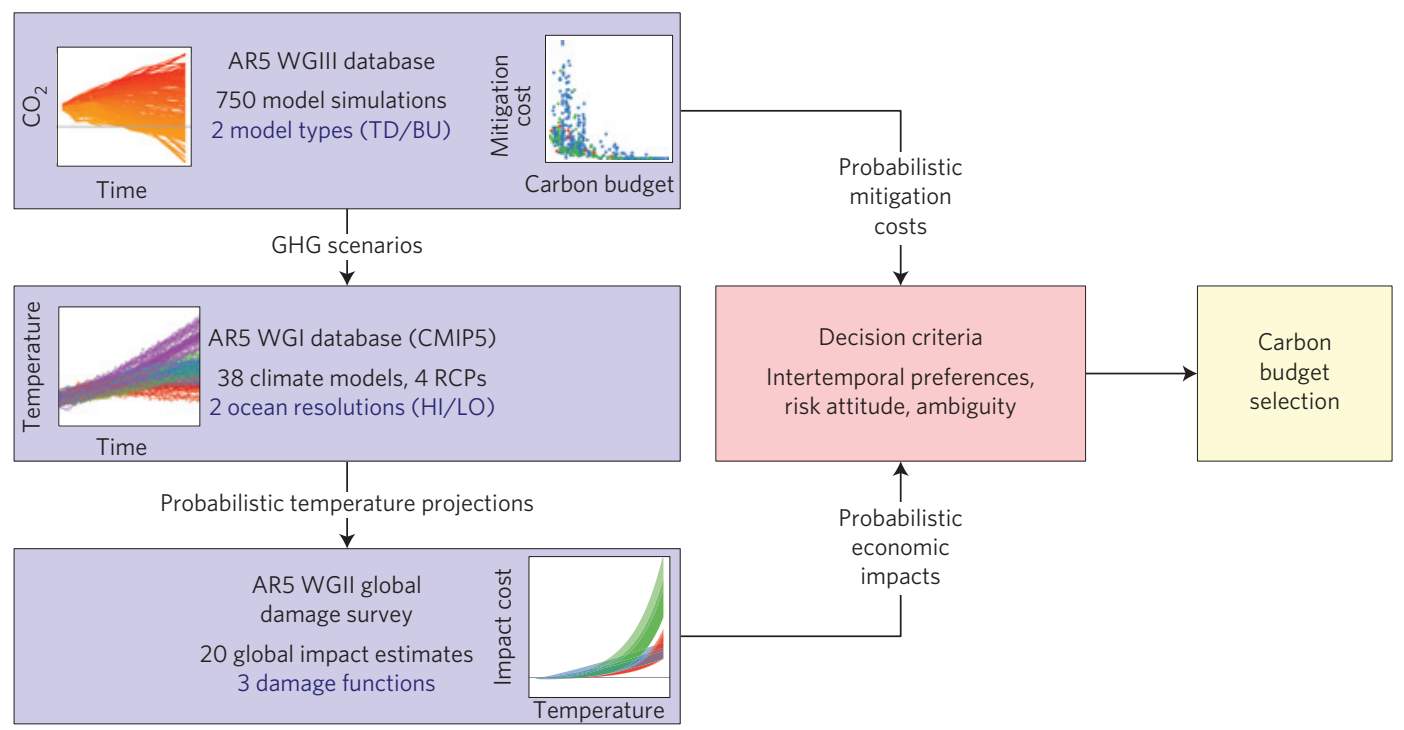

Figure 1 | Diagram illustrating the methodology. Greenhouse gas emission scenarios and mitigation costs are extracted from the dataset used by Working Group III (WGIII) of AR5. Temperature projections are computed from the fifth phase of the Coupled Model Inter-comparison Project (CMIP5) runs of the working group I (WGI) outcomes. Global economic impacts are generated on the basis of the impact reviews proposed in the Fifth Assessment Report, working group II (WGII). Finally, carbon budgets are selected from a set of decision rules and preferences. For more details, refer to Methods.

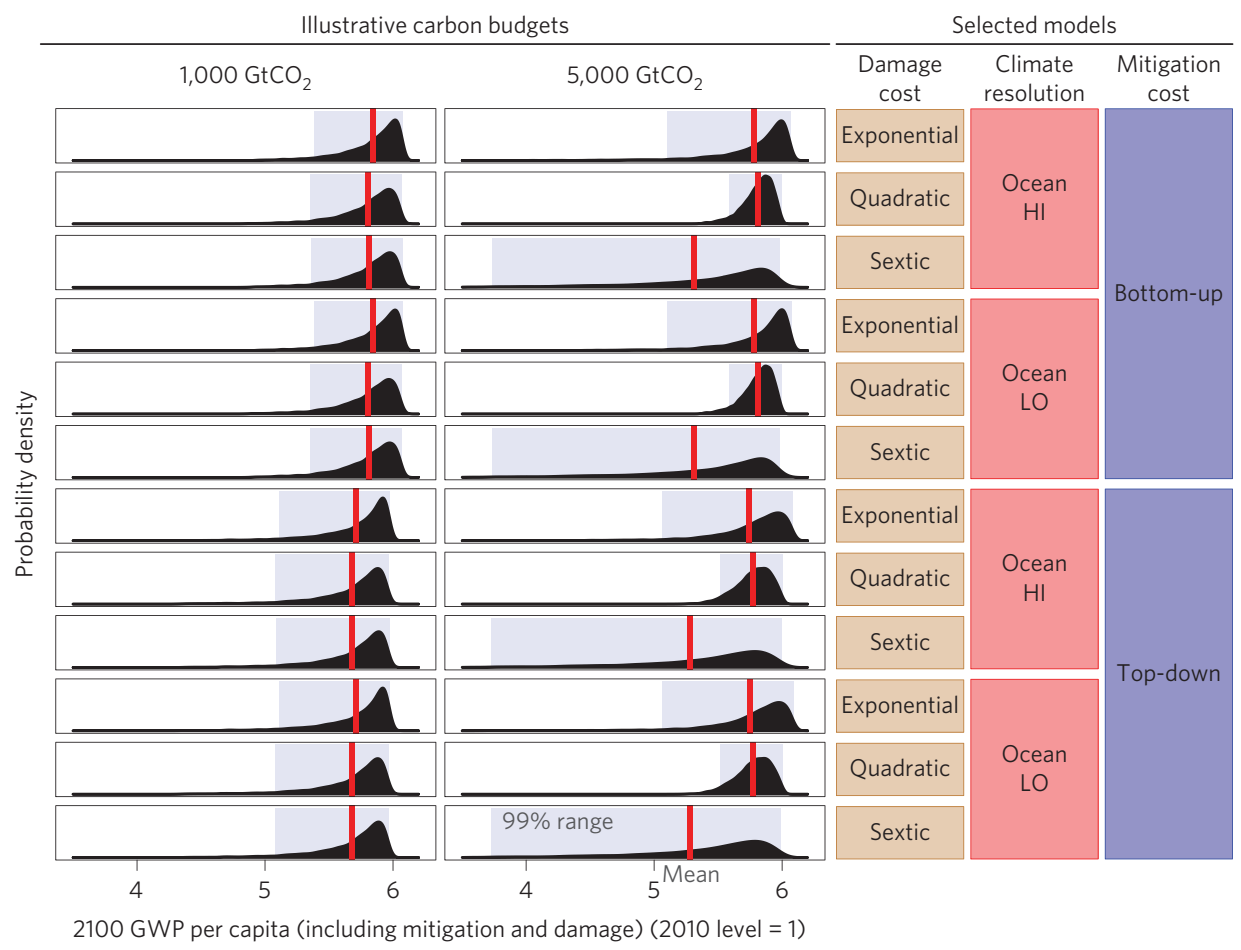

Figure 2 | Influence of carbon budgets, model and state uncertainties on the distribution of the gross world product (GWP) per capita in 2100. The GWP per capita, net of mitigation and damage costs, is expressed relative to its 2010 level. Results are provided for two illustrative and well-distanced $\mathrm{CO}_{2}$ carbon budgets (cumulative emissions over the period 2010-2100 of 1,000 and 5,000 $\mathrm{GtCO}_{2}$ ). The distribution means are indicated by a red line, and $99 \%$ percentile ranges in blue shades. $\mathrm{HI}$ and LO stand for high and low resolution, respectively.

The second step is to generate probabilistic temperature projections for each emission scenario. The projections are generated by a probabilistic climate model based on a reduced complexity climate mode ${ }^{23}$ calibrated to emulate the temperature projections from the fifth phase of the Coupled Model Intercomparison Project (CMIP5; ref. 24). In addition to the whole set of CMIP5 data set, we distinguish two classes of climate models-high and low resolution in the modelling of ocean dynamics-which give significant difference in temperature projection at the end of the twenty-first century (see Supplementary Figs 5 and 6). This allows us to account for climate-model uncertainty.

Finally, we link temperature increases to global economic impact using data reported in the AR5 WGII (ref. 3). These estimates do not include all the potential damage from climate change ${ }^{25,26}$, 

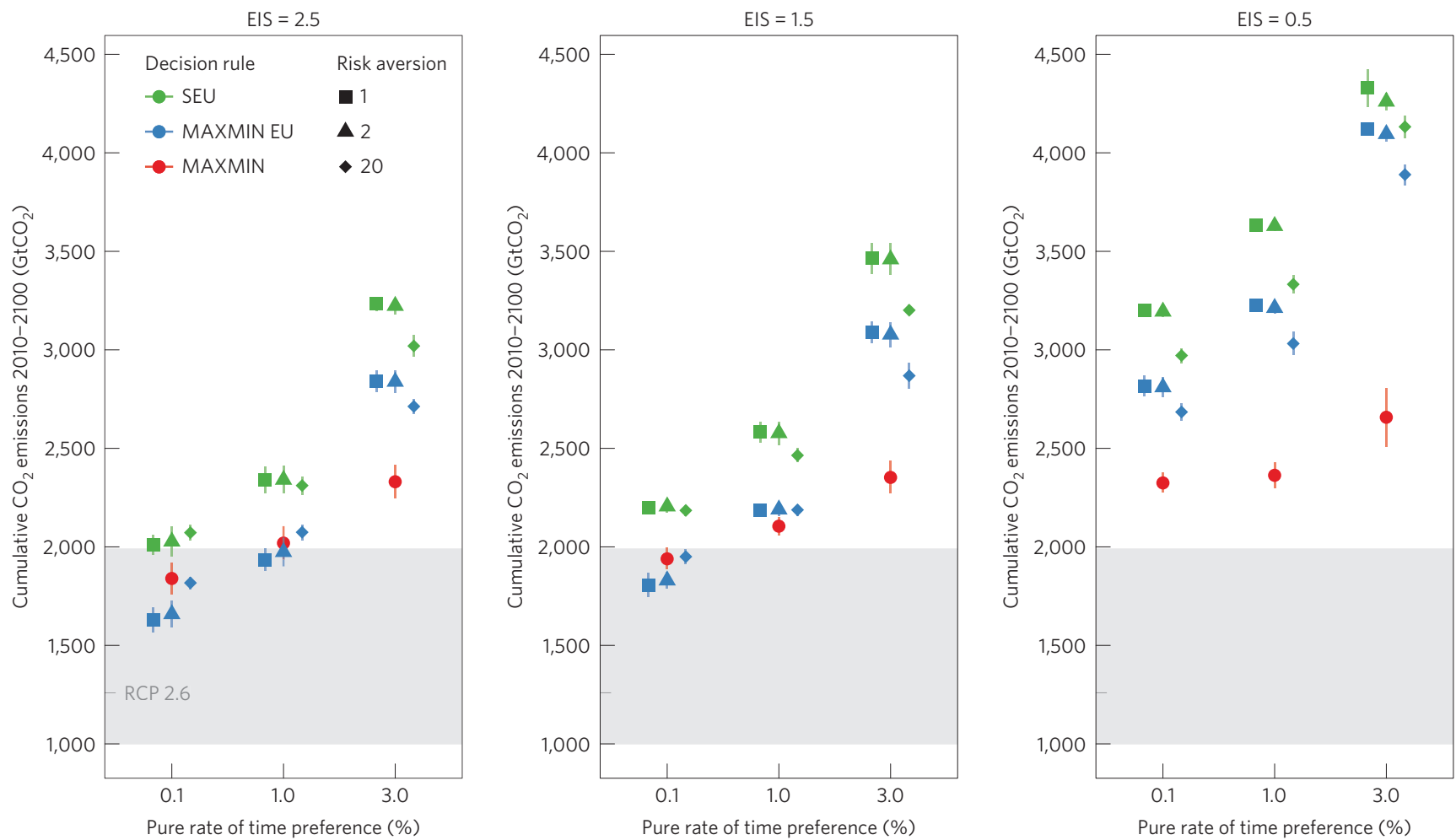

Figure 3 | Selected carbon budgets according to three decision-making criteria: relative risk aversion, consumption smoothing and pure rate of time preference. The shaded area represents the carbon budget range compatible with a radiative forcing of $2.6 \mathrm{~W} \mathrm{~m}^{-2}$, according to Table SPM. 3 of the IPCC AR5 from WGI (ref. 2). The Representative Concentration Pathway 2.6 carbon budget (RCP2.6) is also shown. The vertical bars represent the estimates of the standard errors using bootstrapping. EIS stands for elasticity of intertemporal substitution. Decision rules are subjective expected utility (SEU), maxmin expected utility (MAXMIN EU) and maxmin (MAXMIN). The legend in a applies to all panels.

but represent the best available knowledge at present and have been used for calculating the social cost of carbon ${ }^{27}$. Given the weak theoretical and empirical consensus on the functional form of the relationship between temperature increase and damage, and the few estimates available, especially for temperatures above $3^{\circ} \mathrm{C}$, we capture model uncertainty by calibrating three damage models of the impact distributions. In addition to the commonly used quadratic specification, we also consider exponential and sextic impact functions (Supplementary Fig. 7).

Each carbon budget can be associated with time-dependent distributions of payoffs (Fig. 2). In the case of a very stringent carbon budget (left panel), climate damage is kept under reasonable control, and both the damage function and the climate resolution model have a negligible impact on the gross world product (GWP). Rather, it is the uncertainty in mitigation costs that has a sizeable impact on the mean, whereas the tails are affected by the choice of the damage model. The right-hand panel presents the results of a significantly higher carbon budget. In this case, model uncertainty regarding damage has huge implications on both the mean and the tails. As this higher budget is consistent with very low mitigation effort, neither model uncertainty nor state uncertainty related to mitigation costs have any significant influence. Climate-model uncertainty seems to play a lesser role, despite its significant impact on late-century temperature increase. Figure 2 also shows how the quadratic damage model, typically employed in most cost-benefit analyses of climate policy ${ }^{13}$, fails entirely in capturing significant fat-tailed impact events, even when considering the uncertainty in climate response.

Given the distributions associated with different carbon budgets, to select climate policies, we use a flexible utility function which allows us to disentangle preferences over time, consumption smoothing, and risk. We specify three decision criteria. The first two criteria are built on the 'classical subjective expected utility' framework $^{5}$. The subjective expected utility criterion (SEU) takes expectations over states of nature and over models (each considered equally likely). The maxmin expected utility criterion (Maxmin EU) combines the expected utility with the maxmin criterion $^{28}$, effectively distinguishing between model and state uncertainty. The carbon budget is selected on the basis of the expected payoff of the most pessimistic model. Finally, we consider the maxmin criterion ${ }^{28}$ in which the decision makers focus on the worst consumption per capita over both world states and models. Additional frameworks have been proposed, but the aforementioned ones are among the best known and provide useful benchmarks ${ }^{7}$.

The selected carbon budgets for the three different decision criteria, as well as for preferences over time, intertemporal substitution and risk, are reported in Fig. 3. Results confirm the relationship between time preference and climate policy-namely, that higher discounting of future payoffs leads to higher $\mathrm{CO}_{2}$ budgets. A similar dynamics occurs with respect to the elasticity of intertemporal substitution (EIS), which measures the propensity to smooth consumption over time from future (richer) generations to current (poorer) generations.

The figure also allows us to quantify the role of preferences over model and state uncertainties. Aversion against model uncertainty (shown by the comparison between SEU and Maxmin EU) leads to significantly more stringent climate policies. Ambiguity about the damage function is the most important driver of model uncertainty, reflecting its highly unknown nature (Supplementary Table 4). Aversion against state uncertainty has an ambiguous impact on climate policy: when the budgets are relatively high (for example, because of high discounting or low EIS), higher risk aversion leads to more stringent carbon budgets, to avoid high climate-change damage. The opposite happens at low budgets, owing to the risks of high mitigation costs. Quantitatively, the impact of risk aversion 
is relatively modest. Finally, maxmin constitutes a limiting case of the maxmin EU: as the focus is exclusively on avoiding the worst outcomes, maxmin never leads to lenient climate policies but it also avoids very stringent ones.

From a policy perspective, the impact of the choice of the decision-making criterion is shown to be as large as that of time discounting and consumption smoothing. The carbon budget across the decision criteria differs from 500 to almost 2,000 $\mathrm{GtCO}_{2}$, a major variation in climate policy stringency. Using the central estimates for the temperature climate response to emissions ${ }^{2}$, this translates into a difference in warming of 0.25 to $1^{\circ} \mathrm{C}$. Carbon budgets compatible with a radiative forcing of $2.6 \mathrm{~W} \mathrm{~m}^{-2}$ - which is associated with the $2{ }^{\circ} \mathrm{C}$ target-are selected with some specifications; however $2{ }^{\circ} \mathrm{C}$ seems to never be optimal under the SEU criterion, which is the one employed in the vast majority of cost-benefit analyses of climate change.

Combining the vast amount of data and information collected in the AR5 with the recent advances in decision theory allows us to quantify the key uncertainties associated with climate change and to propose a methodology for selecting global climate policies under different preference structures. We show that aversion to both model and state uncertainty has a major impact on the selection of policies, as a result of the scarcity of knowledge that is still prevalent in the literature on economic assessments of climate change ${ }^{29}$. Uncertainties reported in the AR5 are likely to be lower bounds for actual uncertainties, and are known to increase when moving from global scales to local scales ${ }^{30}$. This might suggest that additional precautions should be taken in devising our collective preferences. Our results point to the need for additional research to understand and better quantify a wider set of climate-change impacts. Similarly, estimates of mitigation costs are still very imprecise, and in many instances fail to include important economic feedback as well as ancillary benefits. Moreover, learning about uncertainties might yield insights on the dynamics of abatement.

So far, uncertainty has exacerbated the polarization in the public debate over climate policies. On the one hand, uncertainty has been interpreted as a reason for limited action on climate, whereas on the other hand, it has been used as a precautionary argument in favour of stringent mitigation. This paper provides one of the few comprehensive approaches to uncertainty quantification in climate change. By helping decision makers to see how their preferences translate into climate policy recommendations, frameworks such as ours can help improve the assessment of climate-change strategies.

\section{Methods}

Methods and any associated references are available in the online version of the paper.

\section{Received 1 February 2015; accepted 12 June 2015; published online 20 July 2015}

\section{References}

1. Kunreuther, H. et al. Risk management and climate change. Nature Clim. Change 3, 447-450 (2013).

2. IPCC Climate Change 2013: The Physical Science Basis (eds Stocker, T. F. et al.) (Cambridge Univ. Press, 2013).

3. IPCC Climate Change 2014: Impacts, Adaptation, and Vulnerability. Part A: Global and Sectoral Aspects (eds Field, C. B. et al.) (Cambridge Univ. Press, 2014).

4. IPCC Climate Change 2014: Mitigation of Climate Change (eds Edenhofer, O. et al.) (Cambridge Univ. Press, 2014).

5. Cerreia-Vioglio, S., Maccheroni, F., Marinacci, M. \& Montrucchio, L. Classical subjective expected utility. Proc. Natl Acad. Sci. USA 110, 6754-6759 (2013).

6. Savage, L. J. The Foundations of Statistics (Dover Publications, 1954).

7. Heal, G. \& Millner, A. Uncertainty and decision making in climate change economics. Rev. Environ. Econ. Policy 8, 120-137 (2014).

8. Ellsberg, D. Risk, ambiguity, and the Savage axioms. Q. J. Econ. 75, 643-669 (1961)
9. Lempert, R. J. Shaping the Next One Hundred Years: New Methods for Quantitative, Long-Term Policy Analysis (Rand, 2003).

10. George, E. I. \& Clyde, M. Model uncertainty. Stat. Sci. 19, 81-94 (2004).

11. Tebaldi, C. \& Knutti, R. The use of the multi-model ensemble in probabilistic climate projections. Phil. Trans. R. Soc. A 365, 2053-2075 (2007)

12. Knutti, R., Furrer, R., Tebaldi, C., Cermak, J. \& Meehl, G. A. Challenges in combining projections from multiple climate models. J. Clim. 23, 2739-2758 (2009)

13. Nordhaus, B. Warming the World: Economic Model of Global Warming (MIT Press, 2000)

14. Millner, A., Dietz, S. \& Heal, G. Scientific ambiguity and climate policy. Environ. Resour. Econ. 55, 21-46 (2013).

15. Ackerman, F., Stanton, E. A. \& Bueno, R. Epstein-Zin utility in dice: Is risk aversion irrelevant to climate policy? Environ. Resour. Econ. 56, 73-84 (2013).

16. Tavoni, M. et al. Post-2020 climate agreements in the major economies assessed in the light of global models. Nature Clim. Change 5, 119-126 (2014).

17. Kriegler, E. et al. The role of technology for achieving climate policy objectives: Overview of the EMF 27 study on global technology and climate policy strategies. Climatic Change 123, 353-367 (2014).

18. Babonneau, F., Haurie, A., Loulou, R. \& Vielle, M. Combining stochastic optimization and Monte Carlo simulation to deal with uncertainties in climate policy assessment. Environ. Model. Assess. 17, 51-76 (2012)

19. Rogeli, J., McCollum, D. L., Reisinger, A., Meinshausen, M. \& Riahi, K. Probabilistic cost estimates for climate change mitigation. Nature 493, 79-83 (2013).

20. Lemoine, D. \& McJeon, H. C. Trapped between two tails: Trading off scientific uncertainties via climate targets. Environ. Res. Lett. 8, 034019 (2013).

21. Meinshausen, M. et al. Greenhouse-gas emission targets for limiting global warming to $2{ }^{\circ} \mathrm{C}$. Nature 458, 1158-1162 (2009).

22. Steinacher, M., Joos, F. \& Stocker, T. F. Allowable carbon emissions lowered by multiple climate targets. Nature 499, 197-201 (2013).

23. Urban, N. M. \& Keller, K. Probabilistic hindcasts and projections of the coupled climate, carbon cycle and Atlantic Meridional Overturning Circulation system: A Bayesian fusion of century-scale observations with a simple model. Tellus $A$ 62, 737-750 (2010).

24. Taylor, K. E., Stouffer, R. J. \& Meehl, G. A. An overview of CMIP5 and the experiment design. Bull. Am. Meteorol. Soc. 93, 485-498 (2012).

25. Ackerman, F., DeCanio, S. J., Howarth, R. B. \& Sheeran, K. Limitations of integrated assessment models of climate change. Climatic Change 95, 297-315 (2009).

26. Pindyck, R. S. Climate change policy: What do the models tell us? J. Econ. Literature 51, 860-872 (2013).

27. Interagency Working Group on Social Cost of Carbon. Technical Update of The Social Cost of Carbon for Regulatory Impact Analysis-Under Executive Order 12866 (United States Government, 2013)

28. Wald, A. Statistical decision functions. Ann. Math. Stat. 20, 165-205 (1949)

29. Houser, T. et al. American Climate Prospectus: Economic Risks in The United States (Rhodium Group, 2014).

30. Knutti, R. \& Sedláček, J. Robustness and uncertainties in the new CMIP5 climate model projections. Nature Clim. Change 3, 369-373 (2013).

\section{Acknowledgements}

We thank K. Keller from the Pennsylvania State University for supplying the climate model and his support. The paper was written while V.B. and M.T. were fellows at the Center for Advanced Studies in the Behavioural Sciences (CASBS) at Stanford University. The research leading to these results has received funding from the Italian Ministry of Education, University and Research and the Italian Ministry of Environment, Land and Sea under the GEMINA project, from the EU FP7 under grant agreement no. 308329 (ADVANCE) and from the European Research Council 336703-RISICO at IEFE, Bocconi University.

\section{Author contributions}

All authors were involved in designing the research and contributed equally to the writing of the manuscript. L.D. performed the scientific computing.

\section{Additional information}

Supplementary information is available in the online version of the paper. Reprints and permissions information is available online at www nature com/reprints.

Correspondence and requests for materials should be addressed to L.D.

\section{Competing financial interests}

The authors declare no competing financial interests. 


\section{Methods}

We propose a method for selecting climate policies which accounts for different preferences for risk, ambiguity and time. We adopt a two-stage subjective expected utility framework ${ }^{5}$ that accounts for both state and model uncertainty. In the context of this paper, 'model uncertainty' refers to the existence of alternative modelling paradigms relating how mitigation costs, the dynamics of the climate system, or economic damage resulting from climate change might respond to climate policies; whereas 'state uncertainty' refers to the probabilistic response (of mitigation costs, temperature, or climate damage) that each of these models produces given a climate policy.

Integrated assessment model data set. The data set is issued from the AR5 scenario database, which has been created for the Integrated Assessment Modeling Consortium (IAMC) and is hosted and maintained by the International Institute for Applied Systems Analysis (IIASA). This database is publicly available and contains outcomes from several model comparison projects, reviewed in the Fifth Assessment Report (AR5) of Working Group III of the Intergovernmental Panel on Climate Change (IPCC). The full description of the database is available in the dedicated website (https://secure.iiasa.ac.at/web-apps/ene/AR5DB) and in Section A.II.10 of the IPCC AR5.

The meta-analysis is carried out with a subset of the AR5 scenario database. We select those long-term scenario-model outcomes that meet the following criteria: model time horizon goes up to the year 2100; mitigation cost estimates are provided; carbon dioxide $\mathrm{CO}_{2}$, methane $\mathrm{CH}_{4}$ and nitrous oxide $\mathrm{N}_{2} \mathrm{O}$ emissions are provided; climate policy category is 'baseline', 'reference', or 'first best'. 'Baseline' scenarios imply no climate policy after 2010, 'reference' scenarios implement a weak policy and current pledges, and 'first best' scenarios have an efficient carbon policy with an immediate target adoption. This leaves us with outcomes from eight integrated assessment models and six model inter-comparisons projects: the Asian Modeling Exercise (AME; ref. 31), the Assessment of Climate Change Mitigation Pathways and Evaluation of the Robustness of Mitigation Cost Estimates (AMPERE) project ${ }^{32}$, the Energy Modeling Forum's Climate Change Control Scenarios (EMF-22) and Global Model Comparison Exercise (EMF-27; ref. 17), the Low climate IMpact scenarios and the Implications of required Tight emissions control Strategies (LIMITS) project ${ }^{16}$ and the Roadmaps towards Sustainable Energy futures (ROSE) project ${ }^{33}$. For each scenario we extract the global emission pathway and the mitigation costs over the century.

Carbon budget. A carbon budget is defined as the cumulative total $\mathrm{CO}_{2}$ emissions over the period 2010-2100. For each scenario, we sum up the world emissions of $\mathrm{CO}_{2}$ from fossil-fuel combustion and industry, and from land-use change. As the database provides the annual emissions every ten years from 2010 to 2100 , the intermediary annual emissions are linearly interpolated (see Supplementary Fig. 1 for an overview of the emission pathways and the carbon budgets from the selected data set).

Mitigation costs. Each scenario is associated with information on mitigation costs. 'Baseline' scenarios have zero mitigation costs. Owing to the different nature of the models, mitigation costs are expressed in three different, but comparable, cost metrics: gross world product (GWP) losses, area under the marginal abatement cost curve, and additional total energy system cost. These costs are converted in $\%$ GWP change from baseline scenario. Supplementary Fig. 2 reports, for each scenario-model outcome, two dimensions: carbon budget and mitigation costs. Carbon budgets are negatively correlated with mitigation costs, in a nonlinear way.

Model categorization is based on a well-documented distinction ${ }^{34}$ between two classes of integrated assessment models: top-down (TD) models, which provide a more accurate description of the macroeconomic feedback, versus bottom-up (BU) models, which better represent the set of mitigation technologies. For the purpose of mitigation costs, TD models generally show higher costs than BU models, but it is not obvious which class of models should be considered as the most accurate.

On the basis of this data, we estimate three piecewise probabilistic models relating, at each time period, carbon budgets and mitigation costs. The procedure, described in the subsequent paragraph, is the same for the three estimated models; what changes are the mitigation cost data used: data coming only from TD models, data coming only from BU models, and the whole data set. First, mitigation costs are clustered in five groups spanning the range of carbon budgets. We fit each cluster data with a Weibull distribution. Second, we estimate, by means of least squares, a relationship between the Weibull distribution parameters and the budgets (the central budget of each cluster is taken as a reference in the fitting). In all cases, each scenario-model outcome is weighted equally. Supplementary Fig. 3 presents the resulting piecewise probabilistic mitigation cost function for the case of the whole data set.

Probabilistic temperature. We use an updated version of a climate model of reduced complexity ${ }^{23}$ to emulate the CMIP5 model ensemble response. This model version is composed of a climate module DOECLIM (ref. 35) and a carbon-cycle model which includes feedbacks from the atmospheric $\mathrm{CO}_{2}$ concentration and temperature ${ }^{36}$. Key geophysical model parameters are estimated from the CMIP5 temperature projections from 2010 to 2100 using a Bayesian inversion technique based on the Markov Chain Monte Carlo (MCMC) algorithm. The estimated climate parameters are the climate sensitivity, the heat vertical diffusivity in the ocean, and the aerosol scaling factor to the total radiative forcing. The carbon-cycle estimated parameters are the carbon fertilization from living plants, the respiration sensitivity related to temperature, and the thermocline carbon transfer rate in the ocean. In addition, initial conditions of atmospheric temperature and $\mathrm{CO}_{2}$ concentration are also estimated.

To perform the MCMC, we constrain the model with the temperature projections for the four Representative Concentration Pathways (RCP2.6, RCP4.5, RCP6.0 and RCP8.5) provided by 38 climate models in the CMIP5 data set to constrain the model. We retain 5,000 equally distant combinations of parameters out of the 3,000,000 in the MCMC to avoid cross-correlation between them. The emulator is able to reproduce the spread of the temperature projections from the CMIP5 data set for the four RCPs (see Supplementary Fig. 4).

It is difficult to distinguish different classes of models from the CMIP5 ensemble as 'there is high confidence that the model performance for global mean surface air temperature (TAS) is high, where the level of confidence is a combination of the level of evidence and the degree of agreement (section on model evaluation in Chapter 9 of the AR5 WGI; ref. 2). Our choice is to split the model outcomes into two classes according to the extent of ocean resolution of the climate model (more or less than 50,000 horizontal grid points). For the RCP4.5 and RCP 6.0, the CMIP5 model provides good agreement, whereas for the more extreme scenario RCP2.6 and RCP 8.5 , the two classes of models diverge slightly after 2070. In these cases, the high-resolution models have a colder atmosphere than the low-resolution models (see Supplementary Fig. 5). Applying the two-sample Welch's $t$-test ${ }^{37}$ on the two subsets of the data, the difference in yearly mean becomes highly significant after 2070 for the RCP2.6 and RCP8.5 (Supplementary Fig. 6).

Once calibrated, the emulator computes probabilistic temperature projections associated with each scenario-model outcome, given information on carbon dioxide emissions, and radiative forcing of other greenhouse gases and of aerosols. The radiative forcing for the non- $\mathrm{CO}_{2}$ greenhouse gases is taken from the database, when available, otherwise it is estimated from the emission levels and their accumulation in the atmosphere. Similarly, the radiative forcing from aerosols is taken from the data set, when available, otherwise it is inferred from the RCP database (available at http://www.iiasa.ac.at/web-apps/tnt/RcpDb). Three sets of projections are produced, one for each of the three probabilistic models (low and high ocean resolution, and the joint set).

Probabilistic impacts of climate change. We use 20 estimates of total economic effects of climate change from the literature reviewed in Table 10.B.1 from Chapter 10 of the IPCC WGII AR5 (ref. 3). These estimates have been calculated using a variety of methods, but they usually aggregate one by one the economic costs accruing in different sectors of both global and local impacts. Each study reports the mean estimates of the economic climate-change damage for a given increase in global mean temperature. Five of the studies also include a measure of the uncertainty surrounding these estimates in the form of the standard deviation (normal distribution) or a confidence interval (skewed distribution). In the case of the skewed distribution, we estimate the parameters of a displaced Gamma distribution matching the reported confidence interval and mean. Given the few data and given that studies only cover temperature increases of up to $4.8^{\circ} \mathrm{C}$, we fit three different probabilistic damage models over the economic climate-change damage data. Let $I_{\mathrm{d}}$ be the economic impacts, expressed in \% of GWP, $T$ be the temperature increase and $\beta_{\mathrm{j}}$ the regression coefficients, then three impact functions are defined. The first is a quadratic impact function $I_{1}(T)=\beta_{1} T+\beta_{2} T^{2}$, as proposed by ref. 38 , which has been used in the DICE integrated assessment model ${ }^{13}$. This function can allow for positive impacts (benefits) at low temperatures. The second is an exponential impact function $I_{2}(T)=\exp \left(-\beta_{3} T^{2}\right)-1$, as introduced by ref. 39 , which excludes the possibility of positive damage (benefits) and which implies greater losses at high warming levels. The third is a sextic impact function $I_{3}(T)=\beta_{4} T^{2}+\beta_{5} T^{6}$, adapted from ref. 40, which implies catastrophic outcomes at extreme temperatures. The economic damage distributions generated by the three models are shown in Supplementary Fig. 7 as probabilistic functions of the temperature increase.

The procedure to estimate the probabilistic relationship between carbon budgets and damage costs is similar to that used in generating the probabilistic mitigation costs models. First, we gather the generated damage costs in five clusters spanning the range of carbon budgets, and we fit each cluster data with a log-normal distribution. Second, we estimate, by means of least squares fit, the relationships between the log-normal distribution parameters and the carbon budgets (using the central point of each cluster as a reference). However, in the case of damage, for each of the carbon budgets we have three temperature probabilistic 
models and, associated with each temperature level, three damage functions. Supplementary Fig. 8 presents the three resulting piecewise probabilistic damage cost functions, for three illustrative years, using temperature projections based on the whole CMIP5 data set model.

Economic projection. We use global projections of population and economic production growth produced by the Organisation for Economic Co-operation and Development (OECD) for the second Shared Socio-economic Pathway (SSP2; ref. 41). The SSP2 describes a 'middle of the road' socio-economic scenario. Let $\bar{Y}$ denote production per capita for each year $t \in T=\{2010, \ldots, 2200\}$, gross of any mitigation or damage cost. At each time period $t$, given each state of the world $s$, and each of the mitigation and damage probabilistic models $m$, the overall economic impacts associated with a carbon budget $c$ is given by the combination of the mitigation cost $M_{t}(c ; s, m)$ and the climate-change damage $D_{t}(c ; s, m)$. Both mitigation and damage are indexed on the combination of models $m$, and $m$ is defined as a triplet selected within the set $\Omega=\{$ \{mitigation-all, mitigation-BU, mitigation-TD $\} \times\{$ climate-all, climate-ocean-lo, climate-ocean-hi $\} \times$ $\{\{$ damage-sextic\}, \{damage-quadratic\}, \{damage-exponential\}\}\}. The classes of model are listed in Supplementary Table 1. As both mitigation and damage losses data are expressed as \% of GWP, we can compute the resulting per capita world production net of mitigation and damage losses.

$$
Y_{t}(c ; s, m)=\bar{Y}_{t} \times\left(1-M_{t}(c ; s, m)\right) \times\left(1-D_{t}(c ; s, m)\right), \quad \forall t \in T
$$

Given that outcomes from the data set end in 2100, we assume that post-2100 mitigation costs decrease linearly, starting from their 2100 level and reaching zero in 2200 , and that post-2100 damage costs remain constant at their 2100 level over the whole twenty-second century. As an illustration, Fig. 2 shows the distributions of $Y_{t}(c ; s, m)$ in 2100, for twelve combinations of models (\{\{mitigation-BU, mitigation-TD $\} \times\{$ climate-ocean-lo, climate-ocean-hi $\} \times\{$ damage-sextic, damage-quadratic, damage-exponential $\}$ ) and for two carbon budgets. Supplementary Fig. 11 provides an intertemporal view of $Y_{t}(c ; s, m)$ for three representative budgets.

Consumption. Not all models included in the data set report the value of global consumption. This is particularly true for BU energy model. As we want to perform our calculation using utility, which is generally a function of consumption, we need to translate GWP into consumption figures. For those models reporting both consumption and GWP, the ratio of the two measures remains constant across scenarios and presents a similar time trend, as depicted in Supplementary Fig. 9. We fit the model mean ratio with a quadratic function and extrapolate it until 2200 (Supplementary Fig. 10). The fitted ratio is 0.741 in 2020, which is consistent with the $26 \%$ world gross saving forecast for the year 2017 by the World Economic Outlook of the International Monetary Fund, slightly increasing over time (to 0.820 in 2200). This procedure allows us to express mitigation and damage losses in terms of consumption losses. In particular, to obtain consumption per capita, we apply the fitted ratio $\zeta_{t}$ to the world net production per capita at each time period.

$$
C_{t}(c ; s, m)=\zeta_{t} Y_{t}(c ; s, m), \quad \forall t \in T
$$

Utility function. To translate consumption per capita into utility, we employ the Epstein-Zin preferences formulation ${ }^{42}$. This formulation allows one to disentangle preferences over time, consumption smoothing and risk. The recursive utility function is

$$
V_{t, \omega}(c ; s, m)=\left[(1-\beta) C_{t}^{1-\rho}(c ; s, m)+\beta\left(\mathbf{E}_{t ; s, m} V_{t+1, \omega}^{1-\alpha}\left(c ; s^{\prime}, m^{\prime}\right)\right)^{\frac{1-\rho}{1-\alpha}}\right]^{\frac{1}{1-\rho}}
$$

where $\mathbf{E}_{t ; s^{\prime}, m^{\prime}}$ is a time-dependent expectation operator over states, $s$, and models, $m \in \omega \subseteq \Omega . \alpha$ and $\beta$ denote the relative risk aversion and the time preference parameter, respectively. $\beta=1 /(1+\delta)$ and $\delta$ is the pure rate of time preference. $\rho$ is the reciprocal of the elasticity of intertemporal substitution (EIS). In the first period $t_{0}=2010$, the equation (1) simplifies to

$$
V_{t_{0}, \omega}(c)=\left[(1-\beta) C_{t_{0}}^{1-\rho}+\beta\left(\mathbf{E}_{s \in S, m \in \omega} V_{t_{0}+1, \omega}^{1-\alpha}(c ; s, m)\right)^{\frac{1-\rho}{1-\alpha}}\right]^{\frac{1}{1-\rho}}
$$

where $C_{t_{0}}$ denotes world consumption in 2010, which is known and independent of the carbon budget, $c$. The expectation operator $\mathbf{E}_{t_{0}}=\mathbf{E}_{s \in S, m \in \omega}$ is applied over the future states of the world $S$ and over a subset of models $\omega$. For $t>t_{0}$, the future state of the world is certain as well as the selected model, so equation (1) is written as

$$
V_{t, \omega}(c ; s, m)=\left[(1-\beta) C_{t}^{1-\rho}(c ; s, m)+\beta\left(V_{t+1, \omega}^{1-\alpha}(c ; s, m)\right)^{\frac{1-\rho}{1-\alpha}}\right]^{\frac{1}{1-\rho}}, \quad \forall t>t_{0}
$$

Starting from the last period 2200, we compute the utility values recursively every year until 2010. We are assuming uncertainty resolves in period 2020; however, we provide a robustness analysis for different learning periods in the Supplementary Information. We specify a utility value of 0 for $t>2,200$.

Decision rules. We build up on the so-called 'classical subjective expected utility' framework ${ }^{5}$. This framework allows us to disentangle two sources of uncertainty: 'model' uncertainty, and 'state' uncertainty. We expand this framework to account for decision makers (DM) with different attitudes towards 'model' and 'state' uncertainty. The decision rules are listed in Supplementary Table 2 and explained hereafter.

Maxmin rule. According to the maxmin criterion, the DM is precautious and discards any probabilistic information. The DM's main objective is to avoid the worst case across both states and models. At each period of time, the DM considers only the worst world consumption per capita over both the states and model dimensions. In this case, the discounted utility $\mathcal{V}_{t}$ is computed as follows

$$
\mathcal{V}_{t}(c)=\left[(1-\beta)\left(\min _{s, m} C_{t}(c ; s, m)\right)^{1-\rho}+\beta \mathcal{V}_{t+1}^{1-\rho}(c)\right]^{\frac{1}{1-\rho}}
$$

Similarly to $V, \mathcal{V}_{t}(c)=0$ for $t>2200$. The DM selects the carbon budget which leads to the highest value for $\mathcal{V}_{t_{0}}(c)$.

Subjective expected utility. According to the subjective expected utility criterion, the DM considered 'model' and 'state' uncertainty as interchangeable and assumes all models are equally valid (as they do not have information to make any better judgement). The expectation operator aggregates over states and model combinations. In this case $\omega=\{\{$ mitigation-all $\} \times\{$ climate-all $\} \times\{$ damage-sextic, damage-quadratic, damage-exponential $\}$ and

$$
V_{t_{0}}(c)=\left[(1-\beta) C_{t_{0}}^{1-\rho}+\beta\left(\mathbf{E}_{s, m} V_{t+1}^{1-\alpha}(c ; s, m)\right)^{\frac{1-\rho}{1-\alpha}}\right]^{\frac{1}{1-\rho}}
$$

This formulation is equivalent to Savage's subjective expected utility decision criterion ${ }^{5}$.

Maxmin expected utility. According to the Maxmin expected utility criterion, 'model' and 'state' uncertainty should be considered differently. In particular, the criterion is dogmatic about which of the models to consider and puts all the weight on the most pessimistic one, whereas for each of the models the expected utility over states of the world is considered.

First, the utility associated with each possible combination of models $\omega=m \in\{\{$ mitigation-BU, mitigation-TD $\} \times\{$ climate-ocean-lo, climate-ocean-hi $\} \times\{$ damage-sextic, damage-quadratic, damage-exponential $\}\}$ is calculated as:

$$
V_{t_{0}, m}(c)=\left[(1-\beta) C_{t_{0}}^{1-\rho}+\beta\left(\mathbf{E}_{s} V_{t+1}^{1-\alpha}(c ; s, m)\right)^{\frac{1-\rho}{1-\alpha}}\right]^{\frac{1}{1-\rho}}
$$

The SEU and Maxmin EU frameworks incorporate, respectively, neutrality and full aversion to the ambiguity related to the specification of the data-generating model. The Maxmin EU criterion focuses on model uncertainty and should not be confused with the Maxmin EU with non-unique prior ${ }^{43}$ which focuses on states of the world.

Carbon budget selection. We generate the policy and damage costs for 100,000 stochastic worlds and 15 nontrivial combinations of model choices,

$\{\{\{$ mitigation-BU $\},\{$ mitigation-TD $\}\} \times\{\{$ climate-ocean-lo $\},\{$ climate-ocean-hi $\}\} \times$ $\{\{$ damage-sextic $\},\{$ damage-quadratic $\},\{$ damage-exponential $\}\}+$ $\{\{$ mitigation-all $\} \times\{$ climate-all $\} \times\{$ ddamage-sextic $\},\{$ damage-quadratic $\}$, \{damage-exponential\}\}\}, for 2,500 carbon budgets within the range 500-6,000 $\mathrm{GtCO}_{2}$. Criteria are computed for different $\delta, \alpha$ and $\rho$. Carbon budgets are selected at the maximum of a function, fitting the generated data by a local polynomial regression. We repeat these steps 5,000 times and estimate the error made in the carbon budget selection. The main results, including the estimated standard errors, are shown in Supplementary Tables 3 and 5.

Parameter specification. $\delta$ represents the pure rate of time preference. We use three values: $0.1 \%, 1 \%$ and $3 \%$, which cover the spectrum of values used in climate policy analysis ${ }^{44,45}$. The default value for EIS is $3 / 2$, with sensitivity to $1 / 2$ and $5 / 2$, as elicited in the literature ${ }^{46,47}$. We allow $\alpha$ to range from 1 to 20 . Bansal and Yaron ${ }^{47}$ suggest a value of around 10

\section{References}

31. Calvin, K. et al. The role of Asia in mitigating climate change: Results from the Asia modeling exercise. Energy Econ. 34, S251-S260 (2012).

32. Riahi, K. et al. Locked into Copenhagen pledges-implications of short-term emission targets for the cost and feasibility of long-term climate goals. Technol. Forecast. Soc. Change 90, 8-23 (2013). 
33. Kriegler, E. et al. Roadmaps Towards Sustainable Energy Futures and Climate Protection: A Synthesis of Results from The RoSE Project 1st edn (Potsdam Institute for Climate Impact Research, 2013); http://www.rose-project.org/ Content/Public/RoSE_REPORT_310513_ES.pdf

34. Fischer, C. \& Morgenstern, R. D. Carbon abatement costs: Why the wide range of estimates? Energy J. 27, 73-86 (2006).

35. Kriegler, E. Imprecise Probability Analysis for Integrated Assessment of Climate Change PhD thesis, Potsdam Univ. (2005).

36. Ricciuto, D. M., Davis, K. J. \& Keller, K. A Bayesian calibration of a simple carbon cycle model: The role of observations in estimating and reducing uncertainty. Glob. Biogeochem. Cycles 22, GB2030 (2008).

37. Welch, B. L. The generalization of 'student's' problem when several different population variances are involved. Biometrika 34, 28-35 (1947).

38. Tol, R. S. Targets for global climate policy: An overview. J. Econ. Dynam. Control 37, 911-928 (2013).

39. Weitzman, M. Handbook of Environmental Accounting 187-207 (Edward Elgar, 2010)
40. Weitzman, M. L. Targets as insurance against catastrophic climate damages. J. Public Econ. Theory 14, 221-244 (2012).

41. Moss, R. H. et al. The next generation of scenarios for climate change research and assessment. Nature 463, 747-756 (2010).

42. Epstein, L. G. \& Zin, S. E. Substitution, risk aversion, and the temporal behavior of consumption and asset returns: A theoretical framework. Econometrica 57, 937-969 (1989).

43. Gilboa, I. \& Schmeidler, D. Maxmin expected utility with non-unique prior. J. Econ. Perspect. 18, 141-153 (1989).

44. Stern, N. The Economics of Climate Change: The Stern Review (Cambridge Univ. Press, 2007).

45. Nordhaus, W. D. \& Weitzman, M. L. A Review of the "Stern Review on the Economics of Climate Change”. J. Econ. Literature 45, 703-724 (2007).

46. Vissing-Jörgensen, A. \& Attanasio, O. P. Stock-market participation, intertemporal substitution, and risk-aversion. Am. Econ. Rev. 93, 383-391 (2003).

47. Bansal, R. \& Yaron, A. Risks for the long run: A potential resolution of asset pricing puzzles. J. Finance 59, 1481-1509 (2004). 